\title{
Appendectomy as a Risk Factor for Bacteremic Biliary Tract Infection Caused by Antibiotic-Resistant Pathogens
}

\author{
Koki Kawanishi, ${ }^{1}$ Jun Kinoshita, ${ }^{1}$ Hiroko Abe, ${ }^{1}$ Tetsuhiro Kakimoto, ${ }^{1}$ \\ Yuko Yasuda, ${ }^{1}$ Takeshi Hara, ${ }^{1}$ and Jun Kato ${ }^{2}$ \\ ${ }^{1}$ Department of Gastroenterology, Wakayama Rosai Hospital, 93-1 Kinomoto, Wakayama City, Wakayama 640-8505, Japan \\ ${ }^{2}$ Second Department of Internal Medicine, Wakayama Medical University, 811 Kimiidera, Wakayama City, \\ Wakayama 641-0012, Japan
}

Correspondence should be addressed to Koki Kawanishi; kawanishi-koki@umin.ac.jp

Received 28 March 2017; Accepted 20 April 2017; Published 15 May 2017

Academic Editor: Heather F. Smith

Copyright (c) 2017 Koki Kawanishi et al. This is an open access article distributed under the Creative Commons Attribution License, which permits unrestricted use, distribution, and reproduction in any medium, provided the original work is properly cited.

\begin{abstract}
Background/Aims. Recent evidence has suggested that appendix plays a pivotal role in the development and preservation of intestinal immune system. The aim of this study is to examine whether prior appendectomy is associated with an increased risk for the development of antibiotic-resistant bacteria in bacteremia from biliary tract infection (BTI). Methods. Charts from 174 consecutive cases of bacteremia derived from BTI were retrospectively reviewed. Using multivariate analysis, independent risk factors for development of antibiotic-resistant bacteria were identified among the clinical parameters, including a history of appendectomy. Results. In total, 221 bacteria strains were identified from 174 BTI events. Of those, 42 antibiotic-resistant bacteria were identified in 34 patients. Multivariate analysis revealed that prior appendectomy (Odds ratio (OR), 3.02; 95\% confidence interval (CI), 1.15-7.87; $p=0.026$ ), antibiotic use within the preceding three months (OR, 3.06; 95\% CI, 1.26-7.64; $p=0.013$ ), and bilioenteric anastomosis or sphincterotomy (OR, 3.77; 95\% CI, 1.51-9.66; $p=0.0046)$ were independent risk factors for antibiotic-resistant bacteria. Conclusions. Prior appendectomy was an independent risk factor for the development of antibiotic-resistant bacteria in bacteremia from BTI.
\end{abstract}

\section{Introduction}

Biliary tract infection (BTI) with bacteremia represents $8-20 \%$ of community-acquired bacteremia in the elderly population and it is the second most common cause of sepsis in those subjects, leading to $10-20 \%$ of mortality $[1,2]$.

Bacteremia in BTI is caused by a wide spectrum of pathogens. In recent years, the prevalence of antibioticresistant pathogens in BTIs has risen steadily $[1,2]$. Infectious conditions caused by those pathogens have also been associated with treatment failure and higher mortality [1, 3]. Several risk factors for the development of antibioticresistant bacteria from BTI have been identified, including healthcare-associated infection, previous hospitalization, previous antibiotic use and bilioenteric anastomosis or sphincterotomy, indwelling biliary drainage, and malignancy $[2,4,5]$. Bacterial pathogens are not always isolated during the course of bacteremia and there is inevitable delay in the identification of susceptibility to antibiotics even in subjects with positive blood culture. Therefore, knowledge of intrinsic resistance patterns of common biliary pathogens is essential for empiric therapy.

Previously, the human appendix has been regarded as a rudimentary part of the intestine. In recent years, however, several studies have suggested its immunological importance for the development and preservation of the intestinal immune system [6]. In addition, the appendix has been shown to have an important interaction with intestinal flora and is believed to work as a "safe house" for commensal gut flora [7]. In this sense, the appendix potentially serves to reinoculate the intestine with normal flora when unbeneficial pathogens arise in the gut [7]. Moreover, it has been reported that prior appendectomy is associated with the recurrence and fulminant course of Clostridium difficile infection $[8,9]$ and recurrence of small intestinal bacterial overgrowth after antibiotic treatment [10]. In this regard, appendectomy may 
be correlated with antibiotic resistance in patients with severe bacterial infections.

The focus of the current study is to analyze whether prior appendectomy is associated with an increased risk of the development of antibiotic-resistant bacteria in bacteremic BTI.

\section{Materials and Methods}

2.1. Patients and Study Design. This retrospective study was conducted at the Wakayama Rosai Hospital in Wakayama, Japan. Charts from 174 consecutive cases of bacteremia derived from BTI treated in the hospital between June 2005 and May 2016 were retrospectively reviewed. Patients with the following criteria were excluded from the study: (i) positive culture for probable skin contaminants (i.e., coagulasenegative Staphylococci, Corynebacterium, Propionibacterium, and Bacillus species) and (ii) bacteremia due to a non-BTI.

Medical records were reviewed and the following information was collected: age, sex, severity of infection (assessed using Pitt bacteremia score), cause of infection, underlying diseases, medication, history of appendectomy, previous antibiotic use (within 90 days), presence of indwelling biliary device, previous hospitalization (within 30 days), and history of bilioenteric anastomosis or sphincterotomy. In addition, 30-day mortality of the patients was examined.

This study was approved by the institutional review board at the Wakayama Rosai Hospital and conforms to the provisions of the Declaration of Helsinki (as revised in Fortaleza, Brazil, October 2013).

2.2. Definitions. Cholecystitis was diagnosed on the basis of clinical presentation of fever, right upper quadrant pain, and findings of ultrasonography or computed tomography. Cholangitis was diagnosed based on the following criteria: (a) presence of fever with upper quadrant pain; (b) radiological (sonographic or computed tomographic) or endoscopic evidence of biliary tract obstruction due to stones or stricture from benign or malignant origin; and (c) laboratory findings of hyperbilirubinemia and an elevated serum alkaline phosphatase level.

The Pitt bacteremia score was used to assess the severities of bacteremic BTIs and was calculated as follows:

(i) Oral temperature: two points for a temperature of $\leq 35^{\circ} \mathrm{C}$ or $\geq 40^{\circ} \mathrm{C}$ or one point for a temperature between 35.1 and $36.0^{\circ} \mathrm{C}$ or between 39.0 and $39.9^{\circ} \mathrm{C}$

(ii) Hypotension: two points for an acute hypotensive event with decreases in systolic $(>30 \mathrm{mmHg})$ and diastolic $(>20 \mathrm{mmHg}$ ) blood pressures, the use of intravenous vasopressor agents, or systolic blood pressure of $<90 \mathrm{mmHg}$

(iii) Receiving mechanical ventilation: two points

(iv) Suffering cardiac arrest: four points

(v) Mental status: being alert, zero points; being disoriented, one point; being stuporous, two points; and being comatose, four points [11]
Antibiotic-resistant bacteria were defined as extendedspectrum $\beta$-lactamase- (ESBL-) producing bacteria, ampicillin-resistant Enterococci and SPACE (Serratia, Pseudomonas, Acinetobacter, Citrobacter, and Enterobacter), in this study according to the previous reports [12-14].

2.3. Microbiological Investigation. Two sets of two $8-10 \mathrm{~mL}$ blood samples were taken from patients who presented with $\geq 38^{\circ} \mathrm{C}$ or who showed clinical signs or symptoms associated with bacteremia. Blood samples were processed in the Oxoid Signal Blood Culture System (Kanto Kagaku, Tokyo, Japan) between June 2005 and October 2011 and processed in an automated BacT/ALERT 3D System (SYSMEX bioMérieux, Tokyo, Japan) between November 2011 and May 2016. The incubation period of blood samples was five days. Positive blood samples were subcultured onto blood agar plates or Brucella blood agar plates. Identification of aerobic and anaerobic bacteria was performed by the WalkAway System (Beckman Coulter) and RapID ANA II (AMCO Inc., Tokyo, Japan), respectively.

2.4. Statistical Analysis. Differences between patients with and without antibiotic-resistant bacteria were determined using the chi-square test and Student's $t$-test as appropriate. Independent risk factors for the detection of antibioticresistant bacteria were identified using multivariate logistic regression analysis that included parameters with $p<0.1$ in univariate analysis. $p$ value $<0.05$ was considered statistically significant and odds ratios (ORs) with 95\% confidence intervals (CIs) were determined. Data were statistically analyzed using JMP version 9 software (SAS Institute, Cary, NC, USA) [15].

\section{Results}

3.1. Clinical Characteristics of Patients. The demographic and clinical features of analyzed patients with bacteremia are summarized in Table 1. A total of 174 (mean age, $70.2 \pm$ $13.9 \mathrm{y}$; male, 106, 61\%) patients with bacteremia due to BTI were identified. The mean value of the Pitt bacteremia score was $1.22 \pm 1.21$. Thirty-two $(18 \%)$ patients had history of appendectomy. Antibiotic use within the preceding three months, indwelling biliary devices, hospitalization within the preceding 30 days, and bilioenteric anastomosis or sphincterotomy were observed in 57 (33\%), 56 (32\%), 51 (29\%), and $42(24 \%)$ patients, respectively. Fourteen patients $(8 \%)$ died within 30 days of admission.

3.2. Microbiological Characteristics. The bacteria strains isolated from the blood cultures are listed in Table 2. In total, 221 bacteria strains were identified from 174 BTI events. Klebsiella species were the most common isolates ( 83 out of $221,38 \%$ ), followed by Escherichia coli (72, 33\%), Enterococcus species (18, 8\%), Streptococcus species (17, 8\%), and Pseudomonas aeruginosa $(10,5 \%)$.

Forty-two antibiotic-resistant bacteria strains were identified in 34 patients (Table 3 ), including 10 Pseudomonas aeruginosa (24\%), 8 Enterobacter species (19\%), seven 
TABLE 1: Characteristics of patients with bacteremia from biliary tract infection.

\begin{tabular}{lc}
\hline Patients & 174 \\
Total & $70.2 \pm 13.9$ \\
Age (mean) & $106(61 \%)$ \\
Male & $1.22 \pm 1.21$ \\
Pitt bacteremia score (mean) & \\
Site of biliary tract infection & $149(86 \%)$ \\
$\quad$ Cholangitis & $25(14 \%)$ \\
$\quad$ Cholecystitis & \\
Cause of biliary tract infection & $116(67 \%)$ \\
$\quad$ No malignancy & $58(33 \%)$ \\
$\quad$ Malignancy & \\
Comorbidity & $47(27 \%)$ \\
$\quad$ Chronic kidney disease & $42(24 \%)$ \\
$\quad$ Diabetes mellitus & $21(12 \%)$ \\
Liver cirrhosis & \\
Medication & $43(25 \%)$ \\
$\quad$ Proton pump inhibitor & $22(13 \%)$ \\
$\quad$ Corticosteroids & $32(18.4 \%)$ \\
Prior appendectomy & $57(33 \%)$ \\
Antibiotic use within the preceding 3 months & $56(32 \%)$ \\
Indwelling biliary device & $51(29 \%)$ \\
Hospitalization within the preceding 30 days & $42(24 \%)$ \\
Bilioenteric anastomosis or sphincterotomy & $14(8 \%)$ \\
30-Day mortality & \\
\hline
\end{tabular}

TABLE 2: Spectrum of bacteria isolated from blood culture in patients with biliary tract infection.

\begin{tabular}{lc}
\hline Bacteria & Total $(N=221)$ \\
\hline Gram-negative organisms & \\
Klebsiella species & $83(38 \%)$ \\
Escherichia coli & $72(33 \%)$ \\
Pseudomonas aeruginosa & $10(5 \%)$ \\
Enterobacter species & $8(4 \%)$ \\
Proteus species & $3(1 \%)$ \\
Citrobacter species & $3(1 \%)$ \\
Serratia species & $1(1 \%)$ \\
Acinetobacter species & $1(1 \%)$ \\
Gram-positive organisms & \\
Enterococcus species & $18(8 \%)$ \\
Streptococcus species & $17(8 \%)$ \\
Staphylococcus species & $2(1 \%)$ \\
Anaerobes & \\
Bacteroides species & $3(1 \%)$ \\
\hline
\end{tabular}

ampicillin-resistant Enterococcus faecium (17\%), 6 ESBLproducing Klebsiella species (14\%), and 5 ESBL-producing Escherichia coli (12\%). Of these, 23 bacteria (55\%) were categorized into the SPACE group.
TABLE 3: Identified antibiotic-resistant bacteria.

\begin{tabular}{lc}
\hline Antibiotic-resistant bacteria & Total $(N=42)$ \\
\hline SPACE & \\
Pseudomonas aeruginosa & $10(24 \%)$ \\
Enterobacter species & $8(19 \%)$ \\
Citrobacter species & $3(7 \%)$ \\
Serratia species & $1(2 \%)$ \\
Acinetobacter species & $1(2 \%)$ \\
Ampicillin-resistant Enterococcus & \\
Enterococcus faecium & $7(17 \%)$ \\
Enterococcus faecalis & $1(2 \%)$ \\
ESBL-producing Klebsiella species & $6(14 \%)$ \\
ESBL-producing Escherichia coli & $5(12 \%)$ \\
\hline
\end{tabular}

SPACE consists of Serratia, Pseudomonas, Acinetobacter, Citrobacter, and Enterobacter. ESBL, extended-spectrum $\beta$-lactamase.

3.3. Risk Factors for Antibiotic-Resistant Bacteria. Differences between patients with and without antibiotic-resistant bacteria are shown in Table 4. Univariate analysis demonstrated that the Pitt bacteremia score, malignancy, prior appendectomy, antibiotic use within the preceding three months, indwelling biliary devices, hospitalization within the preceding 30 days, and bilioenteric anastomosis or sphincterotomy were statistically significant.

Multivariate analysis revealed that prior appendectomy (OR, 3.02; CI, 1.15-7.88; $p=0.026$ ), antibiotic use within the preceding three months (OR, 3.06; 95\% CI, 1.26-7.64; $p=0.013)$, and bilioenteric anastomosis or sphincterotomy (OR, 3.77; CI, 1.51-9.66; $p=0.0046$ ) were the independent risk factors for antibiotic-resistant bacteria (Table 5). Of the 34 patients with antibiotic-resistant bacteremia, those with prior appendectomy were significantly younger than those without $(66.4 \pm 2.8$ years old versus $73.9 \pm 2.1$ years old, $p=0.039$ ), suggesting that appendectomy contributes to the development of antibiotic-resistant bacteria in relatively younger subjects.

\section{Discussion}

The present study demonstrates that history of appendectomy is a significant risk factor for development of antibioticresistant pathogens in bacteremia patients resulting from BTIs. Of the 174 patients examined, 34 developed bacteremia with antibiotic-resistant pathogens, 12 (35\%) of which had a history of appendectomy, whereas only 20 (14\%) of the remaining 140 subjects had the history. Because the lifetime risk of appendectomy has been reported to be $17.6 \%$ in the national hospital discharge survey [16], our cohort appeared to be common and hence the results could be generalized. In addition to prior appendectomy, antibiotic use within the preceding three months and bilioenteric anastomosis or sphincterotomy were found to be independent risk factors. These findings are consistent with those of previous studies $[2,4,5]$ and also suggest generalizability of our study.

The immunological functions of the appendix may work for prevention of prevalence of antibiotic-resistant bacteria. 
TABLE 4: Differences between patients with and without antibiotic-resistant bacteria.

\begin{tabular}{|c|c|c|c|}
\hline & \multicolumn{2}{|c|}{ Antibiotic-resistant bacteria } & \multirow[b]{2}{*}{$p$ value } \\
\hline & $(+)(N=34)$ & $(-)(N=140)$ & \\
\hline Age (mean) & 71 & 69 & 0.67 \\
\hline Male & $19(56 \%)$ & $87(62 \%)$ & 0.50 \\
\hline Pitt bacteremia score (mean) & 1.65 & 1.11 & 0.021 \\
\hline \multicolumn{4}{|l|}{ Site of biliary tract infection } \\
\hline Cholangitis & $29(85 \%)$ & $120(86 \%)$ & 0.95 \\
\hline Cholecystitis & $5(15 \%)$ & $20(14 \%)$ & 0.95 \\
\hline Cause of biliary tract infection & & & 0.058 \\
\hline No malignancy & $18(53 \%)$ & $98(70 \%)$ & \\
\hline Malignancy & $16(47 \%)$ & $42(30 \%)$ & \\
\hline \multicolumn{4}{|l|}{ Comorbidity } \\
\hline Chronic kidney disease & $10(29 \%)$ & $37(26 \%)$ & 0.73 \\
\hline Diabetes mellitus & $8(24 \%)$ & $34(24 \%)$ & 0.92 \\
\hline Liver cirrhosis & $2(6 \%)$ & $19(14 \%)$ & 0.21 \\
\hline \multicolumn{4}{|l|}{ Medication } \\
\hline Proton pump inhibitor & $9(26 \%)$ & $34(24 \%)$ & 0.80 \\
\hline Corticosteroids & $5(15 \%)$ & $17(12 \%)$ & 0.69 \\
\hline Prior appendectomy & $12(35 \%)$ & $20(14 \%)$ & 0.0046 \\
\hline Antibiotic use within the preceding 3 months & $19(56 \%)$ & $38(27 \%)$ & 0.0014 \\
\hline Indwelling biliary device & $15(44 \%)$ & $41(29 \%)$ & 0.090 \\
\hline Hospitalization within the preceding 30 days & $15(44 \%)$ & $36(26 \%)$ & 0.035 \\
\hline Bilioenteric anastomosis or sphincterotomy & $14(41 \%)$ & $28(20 \%)$ & 0.0096 \\
\hline 30-Day mortality & $4(12 \%)$ & $10(7 \%)$ & 0.79 \\
\hline
\end{tabular}

TABLE 5: Risk factors for antibiotic-resistant bacteria (multivariate analysis).

\begin{tabular}{lcc}
\hline & $\begin{array}{c}\text { Odds ratio } \\
(95 \% \text { confidence interval) }\end{array}$ & $p$ \\
\hline $\begin{array}{l}\text { Appendectomy } \\
\text { Pitt bacteremia score (per 1) }\end{array}$ & $1.31(0.54-1.48-7.87)$ & $0.026^{*}$ \\
$\begin{array}{l}\text { Malignancy } \\
\text { Antibiotic use within the } \\
\text { preceding 3 months }\end{array}$ & $3.06(0.60-3.56)$ & 0.40 \\
$\begin{array}{l}\text { Indwelling biliary device } \\
\text { Hospitalization within the } \\
\text { preceding 30 days }\end{array}$ & $1.34(0.55-3.19)$ & 0.51 \\
$\begin{array}{l}\text { Bilioenteric anastomosis or } \\
\text { sphincterotomy }\end{array}$ & $3.75(0.99-6.09)$ & 0.051 \\
\hline
\end{tabular}

* means statistically significant.

The human appendix is associated with the highest concentration of gut-associated lymphoid tissue (GALT) in the gut [17]. GALT is a major source of plasma cell precursors, which migrate specifically to the intestinal lamina propria, and it is the site of initial $\mathrm{CD}^{+}{ }^{+} \mathrm{T}$ cells activation [18]. Moreover, GALT are responsible for the generation of IgAsecreting cells and decreased colonic IgA $(+)$ cells correlate with altered fecal microbiota composition [19]. In this regard, it has been noted that appendectomy markedly reduces the total intestinal plasma cells and immunoglobulins in the animal model [20]. In addition, the appendix is regarded as a natural immunologic barrier to bacterial translocation from the colon to the small bowel [10]. Thus, loss of the appendix by appendectomy may impair intestinal immunity and increase the risk of development of antibiotic-resistant bacteria of which virulence is usually attenuated.

Other functions of the appendix associated with intestinal flora may also be correlated with the results of the current study. The human appendix contains a wealth of microbes, including members of 15 phyla, which constitute more than $98 \%$ of the normal colonic microbiome [21]. This indicates that the appendix possesses a microbial diversity sufficient to reconstitute the microbiome of the intestine. To reinforce this concept, biofilms were found to be most abundant in the appendix [22]. Since biofilms are protective of bacteria, fragments of biofilms routinely shed from the appendix could serve as seeds for inoculation of the intestine with a normal microbial flora [23]. In this sense, the appendix, a potential harbor of normal flora for the intestines, can aid in the repopulation of commensal bacteria and lessen the uncontrollable overgrowth of abnormal, including antibioticresistant, bacteria in the intestine $[24,25]$. This explanation matches the fact that the location of the appendix is secluded from the fecal stream, which presumably affords some protection from pathogenic organisms in gastrointestinal tract.

The present study indicates that appendectomy contributes to the development of antibiotic-resistant bacteria 
in relatively younger subjects with BTIs. Older subjects generally have more comorbid diseases and are relatively immunocompromised. Therefore, appendectomy may no longer affect their immunological defense. Alternatively, the functions of the appendix may attenuate in older subjects because the number of lymphoid follicles and length and diameter of the appendix are known to degenerate with age [26]. In clinical practice of bacteremia from BTI, a history of appendectomy should be noted, particularly in younger subjects.

Considering these facts, the appendix should no longer be regarded as a rudimentary organ and we should carefully decide whether appendectomy should always be indicated for appendicitis. Efficacy of conservative treatment with antibiotics on appendicitis is still controversial. A meta-analysis of randomized controlled trials showed that antibiotics were both effective and safe as primary treatment for patients with uncomplicated acute appendicitis [27]. The largest randomized clinical trial published in 2015 concluded that, among patients with uncomplicated appendicitis, antibiotic treatment did not meet the noninferiority compared with appendectomy [28], although over $70 \%$ of patients of the antibiotics group did not show recurrence within one year. The use of appendectomy or not for appendicitis should be determined by longer follow-up studies. Our results may be considered when making the choice of first-line therapy.

Based on the results of the current study, the recommended first choice of antibiotics for patients with bacteremia from BTI could be based on the presence or absence of identified risk factors including a history of appendectomy. In the Tokyo Guidelines [2], broad-spectrum antibiotics such as tazobactam/piperacillin, cefepime, cefozopran, ceftazidime, aztreonam \pm metronidazole, and carbapenem are recommended for severe BTI and healthcare-associated BTI, whereas sulbactam/ampicillin, sulbactam/cefoperazone, cefazolin \pm metronidazole, cefmetazole, and new quinolone \pm metronidazole are indicated for others. For patients with the risks identified in this study, however, carbapenem plus vancomycin may be the first choice because the combination covers Pseudomonas aeruginosa, ampicillin-resistant Enterococcus, and ESBL-producing pathogens which were most frequently identified antibiotic-resistant pathogens in this study.

We demonstrated that history of appendectomy is a risk of development of antibiotic-resistant bacteria in patients with bacteremia from BTI. The majority of BTI patients, however, showed negative results in blood culture. The causative pathogens in these blood culture-negative BTI patients may also be different according to the risk factors including history of appendectomy. Although it may be difficult to verify the hypothesis due to difficulty in bacteria detection, bile culture could be an alternative, particularly in cases of cholangitis. Moreover, it is potentially useful to investigate whether causative microorganisms in a variety of infectious diseases other than BTI differ between patients with and without a history of appendectomy. Further studies are expected for these clinically relevant issues.

There are limitations to this study. First, the number of the examined patients was relatively small and all subjects were
Japanese. Reproducibility should be confirmed with larger cohorts including other ethnic populations. Another drawback, due to the study being retrospective, is the possibility of missing relevant clinical parameters and inclusion of bias caused by missed blood culture at appropriate timing.

In conclusion, prior appendectomy was identified as an independent risk factor for antibiotic-resistant bacteria in BTIs. Before the choice of antibiotics, interviews regarding history of appendectomy are mandatory as well as other risk factors, including antibiotic use within the preceding three months and bilioenteric anastomosis or sphincterotomy.

\section{Abbreviations \\ BTI: Biliary tract infection \\ ESBL: $\quad$ Extended-spectrum $\beta$-lactamase \\ GALT: Gut-associated lymphoid tissue \\ SPACE: Serratia, Pseudomonas, Acinetobacter, Citrobacter, and Enterobacter.}

\section{Conflicts of Interest}

The authors have no conflicts of interest to declare in relation to this study.

\section{Authors' Contributions}

All authors contributed significantly towards the completion of this study. Koki Kawanishi and Jun Kato contributed to study design and execution, data analysis, and manuscript writing; Jun Kinoshita, Hiroko Abe, Tetsuhiro Kakimoto, Yuko Yasuda, and Takeshi Hara contributed to study execution and data analysis.

\section{References}

[1] M. Melzer, R. Toner, S. Lacey, E. Bettany, and G. Rait, "Biliary tract infection and bacteraemia: Presentation, structural abnormalities, causative organisms and clinical outcomes," Postgraduate Medical Journal, vol. 83, no. 986, pp. 773-776, 2007.

[2] T. Takada, S. M. Strasberg, J. S. Solomkin, H. A. Pitt, H. Gomi, M. Yoshida et al., "TG13: updated tokyo guidelines for the management of acute cholangitis and cholecystitis," Journal of Hepatobiliary Pancreatic Sciences, vol. 20, pp. 1-7, 2013.

[3] J. C. Goo, M. Hyuk Seong, Y. Kwang Shim et al., "Extended spectrum- $\beta$-lactamase or carbapenemase producing bacteria isolated from patients with acute cholangitis," Clinical Endoscopy, vol. 45, no. 2, pp. 155-160, 2012.

[4] Y. K. Sung, J. K. Lee, K. H. Lee, K. T. Lee, and C.-I. Kang, "The clinical epidemiology and outcomes of bacteremic biliary tract infections caused by antimicrobial-resistant pathogens," American Journal of Gastroenterology, vol. 107, no. 3, pp. 473483, 2012.

[5] C. Royo-Cebrecos, C. Gudiol, J. García et al., "Characteristics, aetiology, antimicrobial resistance and outcomes of bacteraemic cholangitis in patients with solid tumours: a prospective cohort study," Journal of Infection, vol. 74, no. 2, pp. 172-178, 5 November 2016.

[6] J.-O. Gebbers and J.-A. Laissue, "Bacterial translocation in the normal human appendix parallels the development of the local 
immune system," Annals of the New York Academy of Sciences, vol. 1029, pp. 337-343, 2004.

[7] I. A. Kooij, S. Sahami, S. L. Meijer, C. J. Buskens, and A. A. Te Velde, "The immunology of the vermiform appendix: a review of the literature," Clinical Experimental Immunology, vol. 186, pp. 1-9, October 2016.

[8] J. Clanton, M. Subichin, K. Drolshagen, T. Daley, and M. S. Firstenberg, "Fulminant Clostridium difficile infection: An association with prior appendectomy?" World Journal of Gastrointestinal Surgery, vol. 5, pp. 233-238, 2013.

[9] F. A. Yong, A. M. Alvarado, H. Wang, J. Tsai, and N. C. Estes, "Appendectomy: A risk factor for colectomy in patients with Clostridium difficile," American Journal of Surgery, vol. 209, no. 3, pp. 532-535, 2015.

[10] E. C. Lauritano, M. Gabrielli, E. Scarpellini et al., "Small intestinal bacterial overgrowth recurrence after antibiotic therapy," American Journal of Gastroenterology, vol. 103, no. 8, pp. 20312035, 2008.

[11] J.-Y. Rhee, K. T. Kwon, H. K. Ki et al., "Scoring systems for prediction of mortality in patients with intensive care unitacquired sepsis: A comparison of the PITT bacteremia score and the acute physiology and chronic health evaluation II scoring systems," Shock, vol. 31, no. 2, pp. 146-150, 2009.

[12] N. Suwantarat and K. C. Carroll, "Epidemiology and molecular characterization of multidrug-resistant Gram-negative bacteria in Southeast Asia," Antimicrobial Resistance Infection Control, vol. 5, Article ID 15, 2016.

[13] F. Hu, D. Zhu, F. Wang, I. Morrissey, J. Wang, and D. Torumkuney, "Results from the Survey of Antibiotic Resistance (SOAR) 2009-11 and 2013-14 in China," Journal of Antimicrobial Chemotherapy, vol. 71, pp. i33-i43, 2016.

[14] A. Kacou-Ndouba, G. Revathi, P. Mwathi et al., "Results from the Survey of Antibiotic Resistance (SOAR) 2011-14 in the Democratic Republic of Congo, Ivory Coast, Republic of Senegal and Kenya," Journal of Antimicrobial Chemotherapy, vol. 71, pp. i21-i31, 2016.

[15] K. Kawanishi, J. Kato, N. Toda et al., "Risk Factors for Aspiration Pneumonia After Endoscopic Hemostasis," Digestive Diseases and Sciences, vol. 61, no. 3, pp. 835-840, 2016.

[16] D. G. Addiss, N. Shaffer, B. S. Fowler, and R. V. Tauxe, "The epidemiology of appendicitis and appendectomy in the United States," The American Journal of Epidemiology, vol. 132, no. 5, pp. 910-925, 1990.

[17] N. L. Sanders, R. R. Bollinger, R. Lee, S. Thomas, and W. Parker, "Appendectomy and Clostridium difficile colitis: Relationships revealed by clinical observations and immunology," World Journal of Gastroenterology, vol. 19, no. 34, pp. 5607-5614, 2013.

[18] M. Matsushita, H. Takakuwa, Y. Matsubayashi, A. Nishio, S. Ikehara, and K. Okazaki, "Appendix is a priming site in the development of ulcerative colitis," World Journal of Gastroenterology, vol. 11, no. 31, pp. 4869-4874, 2005.

[19] K. Masahata, E. Umemoto, and H. Kayama, "Generation of colonic IgA-secreting cells in the caecal patch," Nature Communications, vol. 5, article 3704, 2014.

[20] J. F. Dasso and M. D. Howell, "Neonatal appendectomy impairs mucosal immunity in rabbits," Cellular Immunology, vol. 181, no. 3, pp. 29-37, 1997.

[21] C. M. Guinane, A. Tadrous, F. Fouhy et al., "Microbial composition of human appendices from patients following appendectomy," mBio, vol. 4, no. 1, 2013.
[22] R. E. Ley, M. Hamady, C. Lozupone et al., "Evolution of mammals and their gut microbes," Science, vol. 320, no. 5883, pp. 1647-1651, 2008.

[23] R. Randal Bollinger, A. S. Barbas, E. L. Bush, S. S. Lin, and W. Parker, "Biofilms in the large bowel suggest an apparent function of the human vermiform appendix," Journal of Theoretical Biology, vol. 249, no. 4, pp. 826-831, 2007.

[24] G. Y. Im, R. J. Modayil, C. T. Lin et al., "The appendix may protect against clostridium difficile recurrence," Clinical Gastroenterology and Hepatology, vol. 9, no. 12, pp. 1072-1077, 2011.

[25] M. Laurin, M. L. Everett, and W. Parker, "The Cecal Appendix: One More Immune Component With a Function Disturbed By Post-Industrial Culture," Anatomical Record, vol. 294, no. 4, pp. 567-579, 2011.

[26] A. Sarkar, A. Saha, S. Roy, S. Pathak, and S. Mandal, "A glimpse towards the vestigiality and fate of human vermiform appendixa histomorphometric study," Journal of Clinical and Diagnostic Research, vol. 9, no. 2, pp. AC11-AC15, 2015.

[27] K. K. Varadhan, K. R. Neal, and D. N. Lobo, "Safety and efficacy of antibiotics compared with appendicectomy for treatment of uncomplicated acute appendicitis: Meta-analysis of randomised controlled trials," BMJ (Online), vol. 344, no. 7855, Article ID e2156, 2012.

[28] P. Salminen, H. Paajanen, T. Rautio et al., "Antibiotic therapy vs appendectomy for treatment of uncomplicated acute appendicitis: The APPAC randomized clinical trial," JAMA - Journal of the American Medical Association, vol. 313, no. 23, pp. 2340-2348, 2015. 


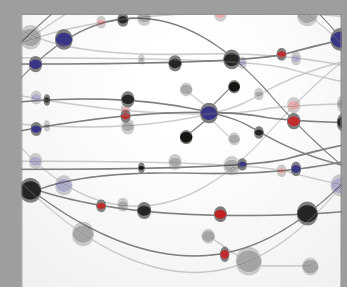

The Scientific World Journal
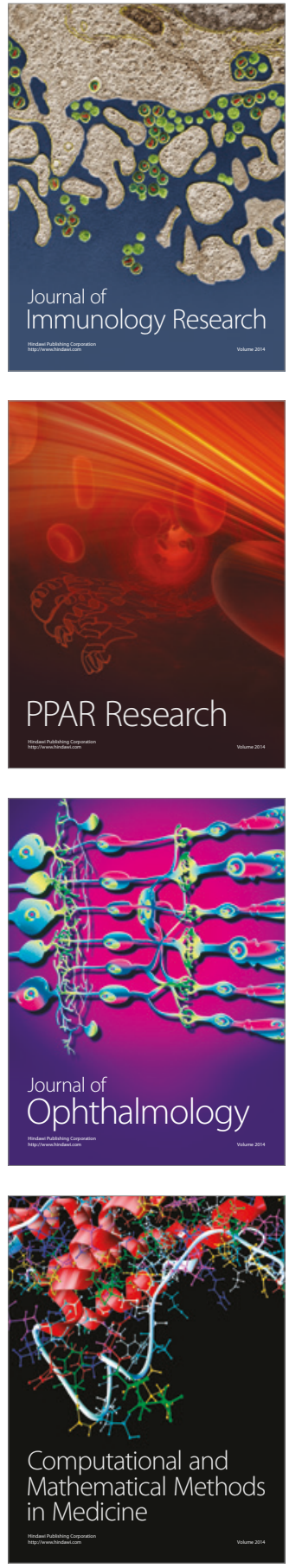

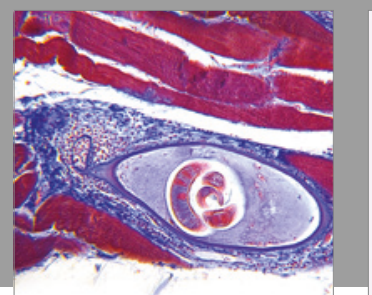

Gastroenterology Research and Practice
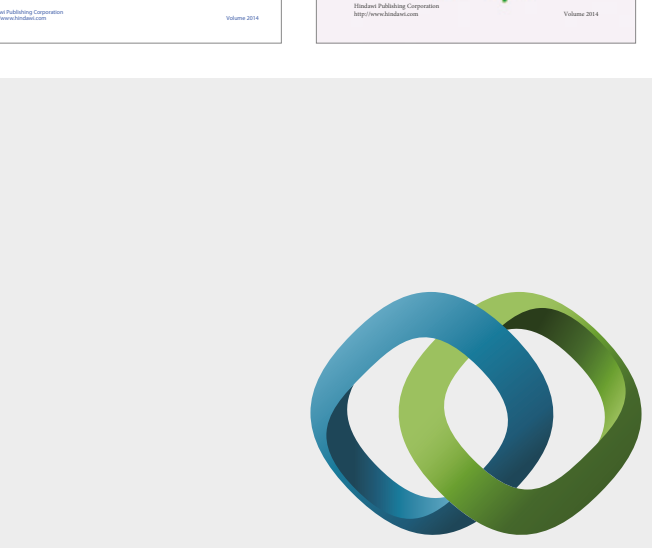

\section{Hindawi}

Submit your manuscripts at

https://www.hindawi.com
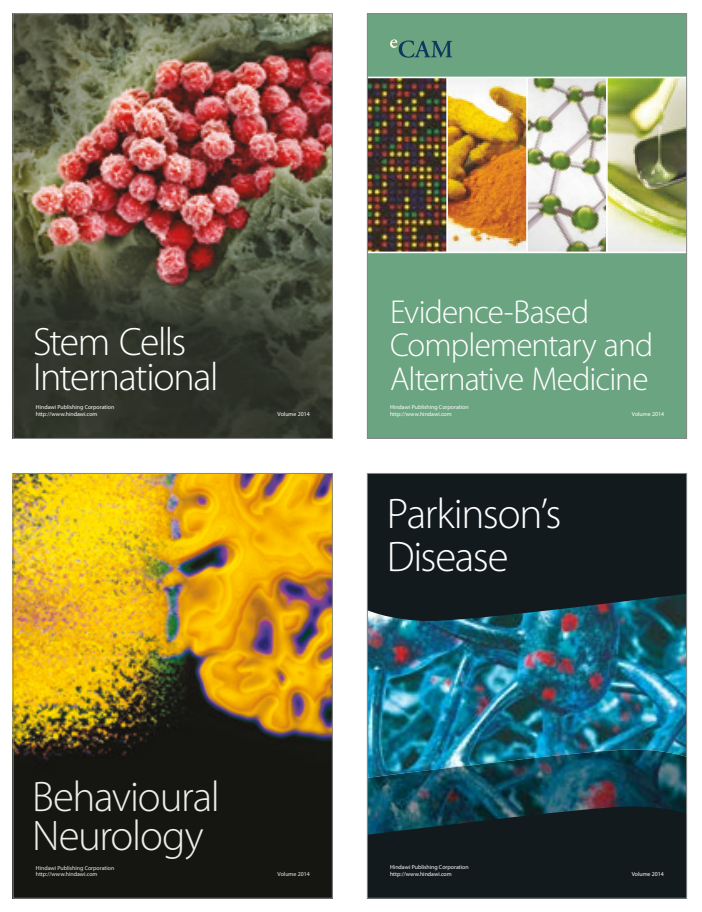
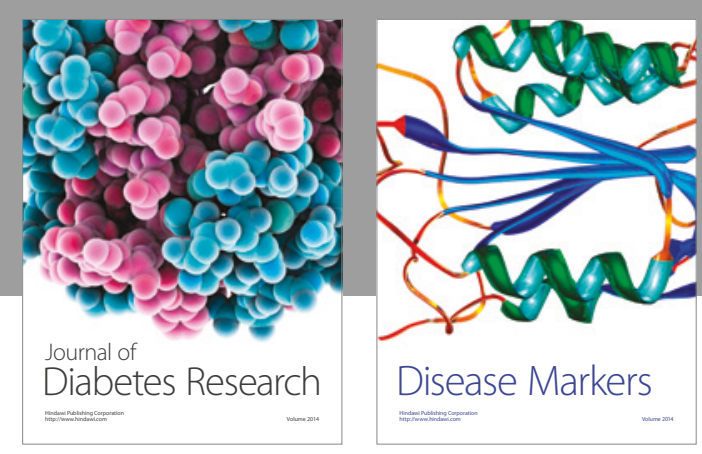

Disease Markers
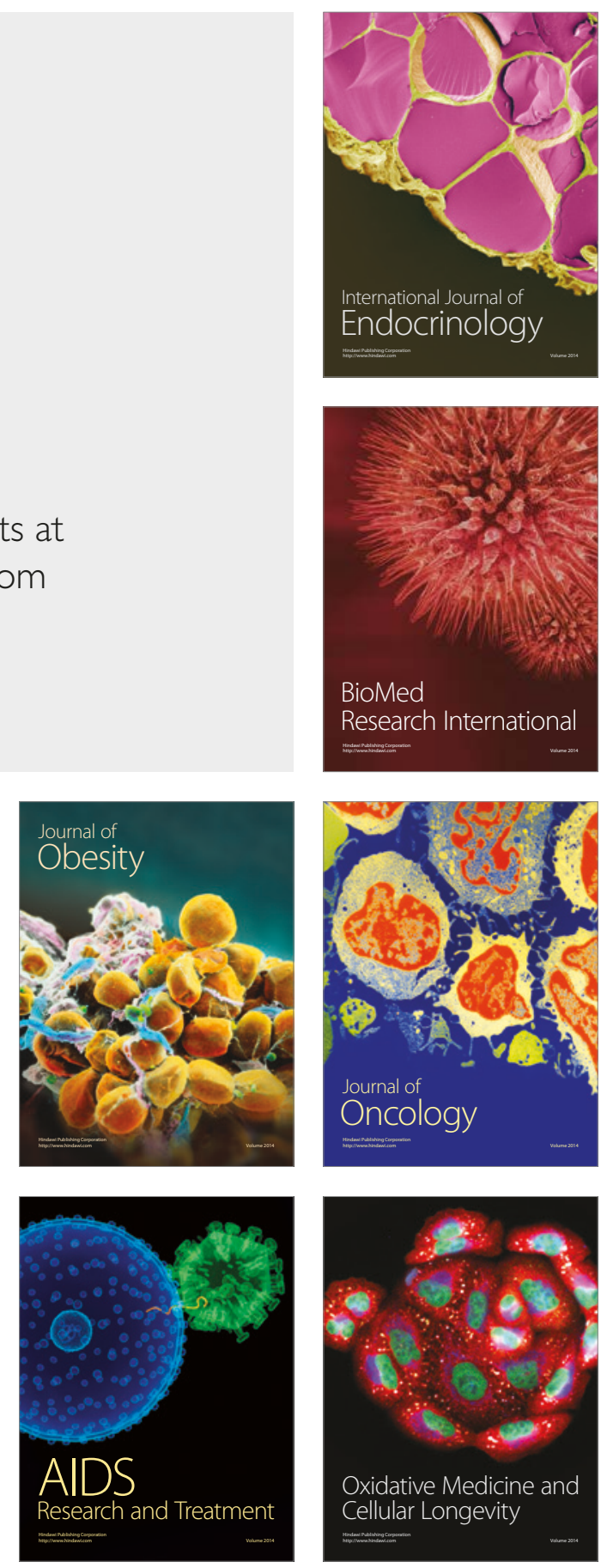